\title{
Isospin splitting of the nucleon mean field
}

\author{
W. Zuo ${ }^{1}$, L.G. Cao ${ }^{2}$, B.A. Li $^{3}$, U. Lombardo ${ }^{2,4}$, C.W. Shen $^{5}$ \\ ${ }^{1}$ Institute of Modern Physics, \\ P.O. Box 31, Lanzhou 730000, China, \\ ${ }^{2}$ LNS-INFN, Via Santa Sofia 44, I-95123 Catania, Italy \\ ${ }^{3}$ Department of Chemistry and Physics, \\ Arkansas State University, \\ State University, AR 72467, USA \\ ${ }^{4}$ Dipartimento di Fisica dell'Universit, \\ Viale Andrea Doria 6, I-95123 Catania, Italy \\ ${ }^{5}$ China Institute of Atomic Energy, \\ P.O.Box 275(18), Beijing 102413, China
}

\begin{abstract}
The isospin splitting of the nucleon mean field is derived from the Brueckner theory extended to asymmetric nuclear matter. The Argonne V18 has been adopted as bare interaction in combination with a microscopic three body force. The isospin splitting of the effective mass is determined from the Brueckner-Hartree-Fock self-energy: It is linear acording to the Lane ansatz and such that $m_{n}^{*}>m_{p}^{*}$ for neutron-rich matter. The symmetry potential is also determined and a comparison is made with the predictions of the Dirac-Brueckner approach and the phenomenological interactions. The theoretical predictions are also compared with the empirical parametrizations of neutron and proton optical-model potentials based on the experimental nucleon-nucleus scattering and the phenomenological ones adopted in transport-model simulations of heavy-ion collisions. The direct contribution of the rearrangement term due to three-body forces to the single particle potential and symmetry potential is discussed.
\end{abstract}

PACS numbers: 26.60.+c,21.30.Fe,21.65.+f

\section{INTRODUCTION}

The study on the role of isospin degree of freedom is in rapid progress in both nuclear physics and nuclear astrophysics. The experimental and theoretical research on isospin physics have received a strong boost because of the construction of more advanced detectors (such as Magnex 11) and new radioactive ion beam facilities (project RIA 2]). A wide range of rich phenomenologies from nuclei far from the $\beta$-stability line to strongly asymmetric compound systems formed in heavy ion collisions (HIC) requires a deep understanding of the isospin dependence of the in-medium nuclear effective interaction in a large range of nucleon density and energy. Among the interesting new physics, a key point is the interplay between the isospin $T=0$ and $T=1$ components of the effective interaction as a function of the isospin asymmetry.

Because of the lack of enough empirical information, the most reliable theoretical tools are the microscopic parameter-free approaches based on realistic nucleon-nucleon (NN) forces fitting the experimental phase shifts of the in-vacuum nucleon-nucleon scattering. One of the most advanced approaches is the nonrelativistic Brueckner theory. Over the last decade, in fact, it has been improved in two aspects: its convergence has been verified at the level of three-body correlations [3] and the empirical saturation point has been reproduced by including microscopic three-body forces (TBF) 4]. Extending the Brueckner calculations to spin and isospin asymmetric nuclear matter impor- tant predictions have been made on physical quantities, including the symmetry energy, and the spin and spinisospin Landau parameters [5, [6].

Intimately related to the effective interaction is the nucleon self-energy, which brings important information on the momentum dependence of nuclear mean field, effective mass and optical potential. In the Brueckner-Hartree-Fock (BHF) approximation the selfenergy takes into account not only the interaction of a nucleon with inert core (pure BHF mean field) but also with core excitations [7, [8]. The latter is crucial for the comparison with the experimental nuclear potential and the optical model potential at low energy.

The isospin splitting of the nucleon self-energy is the main topic of the present investigation. It has been calculated in wide ranges of isospin asymmetry, density and energy for the sake of application in transport simulations of HIC as well as for structure calculations close to the neutron and proton drip lines. Despite some results existing in the literature since long time, the present work has been stimulated not only by the new opportunity that we can now study the effects of threebody forces in the Brueckner theory, but also by the appearance of relativistic Dirac-Brueckner-Hartree-Fock (DBHF) calculations 9, 10]. Consistent microscopic predictions could provide strong constraints for phenomenological approaches, which are still affected by large uncertainties. These constraints may lead to the need of new parametrizations of the Skyrme-like interactions for the calculations far from the beta-stability line. 
The isovector part of the neutron and proton single particle (s.p.) potentials, i.e., the symmetry potential, is one of the basic inputs of the transport models for the collisions of radioactive nuclei. In general, the shape of the symmetry energy as a function of density is determined simultaneously by both the momentum and density dependence of the symmetry potential 11], therefore the determination of the momentum dependence of the symmetry potential is crucial for constraining the high density behavior of symmetry energy. In the earlier dynamical simulations of HIC, the momentum dependence of the symmetry potential was seldom taken into account. Only recently, Das et al. 12] has proposed some simple phenomenological parametrizations for the momentum dependence of the symmetry potential which have been adopted in the dynamical simulations of HIC by Li et al. 13, 14, 15] where it is shown that the experimental observables such as the neutron-proton differential flow, the isospin fractionation and the $\pi^{-} / \pi^{+}$ratio etc., are quite sensitive to the momentum dependence of the symmetry potential. Microscopically, the proton and neutron s.p. potentials and their isospin dependence have been studied in Ref. [5, 8] within the BHF approach. However, the momentum and density dependence of the symmetry potential was not discussed in our previous investigations [5, [8].

In the present paper, we will concentrate on the discussion of the isospin splitting of the effective mass and the density and momentum dependence of the symmetry potential, based on the BHF approximation [8]. Especially we shall compare our microscopic symmetry potential with the phenomenological ones of Ref. 12 and the predictions of the Dirac-Brueckner method 9, 10]. The present paper is arranged as follows. In Sec. II we present a brief introduction of the Brueckner-Bethe-Goldstone (BBG) theory for Gmatrix with a microscopic TBF, including self-energy and effective mass of protons and neutrons. The numerical results for the symmetry potential are reported and discussed in Sec. III in comparison with other model predictions. Summary and conclusions are drawn in Sec. IV.

\section{SELF-CONSISTENT BHF APPROACH INCLUDING A THREE-BODY FORCE}

\section{A. BBG equation}

The Brueckner theory and its extension to include TBFs are described elsewhere [4]. Here we simply give a brief review for completeness. The starting point of the $\mathrm{BHF}$ approach is the reaction $G$-matrix, which satisfies the following isospin dependent Bethe-Goldstone (BG) equation,

$$
\begin{aligned}
G(\rho, \beta, \omega) & = \\
v_{N N} & +v_{N N} \sum_{k_{1} k_{2}} \frac{\left|k_{1} k_{2}\right\rangle Q\left(k_{1}, k_{2}\right)\left\langle k_{1} k_{2}\right|}{\omega-\epsilon\left(k_{1}\right)-\epsilon\left(k_{2}\right)} G(\rho, \beta, \omega),
\end{aligned}
$$

where $k_{i} \equiv\left(\vec{k}_{i}, \sigma_{1}, \tau_{i}\right)$, denotes the single particle momentum, the $z$-component of spin and isospin, respectively. $v_{N N}$ is the realistic nucleon-nucleon $(N N)$ interaction, $\omega$ is the starting energy. The asymmetry parameter is defined as $\beta=\left(\rho_{n}-\rho_{p}\right) / \rho$, where $\rho, \rho_{n}$, and $\rho_{p}$ denote the total, neutron and proton number densities, respectively. For the $N N$ interaction, we adopt the Argonne $V_{18}$ two-body interaction 16 plus a microscopic three-body force (TBF) [17]. The TBF is constructed by using the meson-exchange current approach [17] and the most important mesons, i.e., $\pi$, $\rho, \sigma$ and $\omega$ have been considered [18]. The parameters of the TBF model have been self-consistently determined so to reproduce the Argonne $V_{18}$ two-body force using the one-boson-exchange potential model [4]. Their values can be found in Ref. [4]. The TBF contains the contributions from different intermediate virtual processes such as virtual nucleon-antinucleon pair excitations, and nucleon resonances ( for details, see Ref. 17]). The TBF effects on the equation of state (EOS) of nuclear matter and its connection to the relativistic effects in the DBHF approach have been reported in Ref. [4].

In solving the BG equation for the $G$-matrix, the continuous choice [7] for the auxiliary potential is adopted since it provides a much faster convergence of the holeline expansion than the gap choice [3]. One advantage of the continuous choice is that the auxiliary potential has the physical meaning of the mean field felt by a nucleon during its propagation between two successive scatterings in nuclear medium [19].

The effect of the TBF is included in the selfconsistent Brueckner procedure along the same lines as in Ref. [17], where an equivalent effective two-body interaction $\tilde{v}$ is constructed by weighting the third particle in the real TBF by means of the defect function. So doing, one avoids the difficulty of solving the full three-body problem. The effective two-body interaction $\tilde{v}$ can expressed in $r$-space as 4

$$
\begin{aligned}
& \left\langle\vec{r}_{1} \vec{r}_{2}|\tilde{v}| \vec{r}_{1}^{\prime} \vec{r}_{2}^{\prime}\right\rangle= \\
& \frac{1}{4} \operatorname{Tr} \sum_{n} \int \mathrm{d} \vec{r}_{3} \mathrm{~d} \vec{r}_{3}^{\prime} \phi_{n}^{*}\left(\vec{r}_{3}^{\prime}\right)\left(1-\eta\left(r_{13}^{\prime}\right)\right)\left(1-\eta\left(r_{23}^{\prime}\right)\right) \\
& \times W_{3}\left(\vec{r}_{1}^{\prime} \vec{r}_{2}^{\prime} \vec{r}_{3}^{\prime} \mid \vec{r}_{1} \vec{r}_{2} \vec{r}_{3}\right) \phi_{n}\left(r_{3}\right)\left(1-\eta\left(r_{13}\right)\right)\left(1-\eta\left(r_{23}\right)\right)
\end{aligned}
$$

where the trace is taken with respect to the spin and isospin of the third nucleon. The function $\eta(r)$ is the defect function. Since the defect function is directly determined by the solution of the BG equation 17], it must be calculated self-consistently with the $G$ matrix and 
the s.p. potential $U(k)[4]$ at each density and isospin asymmetry. It is evident from Eq.(2) that the effective force $\tilde{v}$ rising from the TBF in nuclear medium is density dependent. A detailed description and justification of the method can be found in Ref. [17].

\section{B. Selfenergy}

In the BHF approximation [8] with the TBF, the selfenergy is made of three terms:

$$
\Sigma(k, \varepsilon)=\Sigma_{b h f}+\Sigma_{c p o l}+\Sigma_{t b f}
$$

where the first term is the HF potential with the $G$ matrix as the effective interaction, the second term is due to the core polarization [7], and the third term stems from the density dependence of the effective force $\tilde{v}$, i.e., the TBF rearrangement term $\tilde{v}$ 20]. The first two terms in the BHF approximation in asymmetric nuclear matter have been discussed elsewhere [8]. In general, the TBF effect on the selfenergy within the BHF framework is twofold. First, it affects the selfenergy via the modification of the $G$-matrix. This effect has been embodied in the BHF selfenergy, i.e., the first two terms in Eq.(3). Second, the density dependence of $\tilde{v}$ will induce an additional contribution, i.e., a rearrangement contribution to the selfenergy (third term in Eq.(3)). The main contribution of the TBF rearrangement can be formally written in the BHF approximation

$$
\Sigma_{t b f}=\frac{1}{2} \sum_{i j}<i j\left|\frac{\delta \tilde{v}}{\delta n_{k}}\right| i j>_{A} n_{i} n_{j}
$$

where $n_{i}$ is the Fermi step function. For most results we present below the selfenergy is calculated in the BHF approximation, and the effect of the TBF is only restricted to the G-matrix via the BBG equation with the two body force and the effective TBF. At the end of Sec. III we will discuss explicit effect of the rearrangement term of the effective three-body force on the self-energy and the symmetry potential.

When calculated on the energy shell the self-energy gives rise to the single nucleon potential. The contribution due to the inert core (BHF) for neutrons and protons is reported in Fig 1 as a function of momentum $k$ for three densities and several isospin asymmetries $\beta \equiv\left(\rho_{n}-\rho_{p}\right) / \rho$. The core polarization term mainly influences the potential at $k<k_{F}[\mathbf{7}]$ and it will be neglected in the discussion of this subsection. In order to explore the isospin effects on the nucleon effective masses (Sec II. C) we split the neutron and proton s.p. potentials into the contributions from the isospin $T=0$ and $T=1$ channels, i.e.,

$$
\begin{gathered}
U_{p}(k, \beta)=U_{p}(k, \beta)_{T=0}+U_{p}(k, \beta)_{T=1} \\
U_{n}(k, \beta)=U_{n}(k, \beta)_{T=0}+U_{n}(k, \beta)_{T=1}
\end{gathered}
$$

The isospin behavior of the neutron or proton s.p. potential is a result of the competition between the $T=0$ and $T=1$ isospin channels. As discussed in Ref. 8, 21] the isospin effect on the EOS of asymmetric nuclear matter is dominated by the isospin $T=0$ component of the NN interaction. In Fig. 2 we present the contributions from the isospin $T=0$ and $T=1$ channels to the proton and neutron s.p. potentials at $k=0$, separately, as a function of $\beta$ with respect to their values in symmetric nuclear matter $(\beta=0)$. It is seen that the variations versus $\beta$ of the $T=0$ components are much larger than the corresponding $T=1$ components, i.e., $3 \sim 5$ times larger, implying that the $\beta$ dependence of the neutron and proton s.p. potentials is determined to a large extent by the $T=0$ component. This is what expected since as increasing the neutron excess, the $T=0$ interaction between two unlike-nucleons ( vanishing between two like-nucleons) becomes stronger for protons and weaker for neutrons. The relatively small deviations of the $T=1$ components of the $U_{n}$ and $U_{p}$ from their common values in symmetric matter is associated to the variations of the Fermi surfaces in neutron-rich matter. It is also seen from the figure that the net contribution of the $T=0$ channels stems almost completely from the $S D$ tensor channel (squares) which is strongly attractive at relatively low energies while the contributions from other $T=0$ channels cancel out each other. This is in agreement with the previous observation for nuclear symmetry energy [21]. As a consequence, at low momenta, the proton s.p. potential becomes more attractive and the neutron one more repulsive going from symmetry nuclear matter $(\beta=0)$ to pure neutron matter $(\beta=1)$, as shown in Fig. 1] According to the experimental data on the phase shifts of nucleon-nucleon scattering, the attraction of the $S D$ channel decreases with energy, so that for a given energy the $T=0$ channel contribution to the splitting $U_{n}-U_{p}$ becomes equal to the $T=1$ channel contribution. As a result the isospin splitting $\left(U_{T=0}-U_{T=1}\right) / \beta$ vanishes for a given value of momentum as marked by the crossing point in Fig. 11 This point is almost independent of both isospin and density. Therefore, the increase of the proton potential depth vs. asymmetry results in an increase of the slope as a function of momentum. This behavior controls the proton and neutron effective mass splitting in neutron rich matter. From the above discussion it may be concluded that the isospin behavior of the momentum dependence of the proton and neutron s.p. potentials which determines the neutron-proton effective mass splitting, is essentially controlled by the tensor component of the NN interaction, or say, by the nature of the NN interaction. The core polarization affects mainly the s.p. potential in the low momentum range below the Fermi surface. It gives a repulsive contribution for both the proton and neutron potentials and weakens the momentum dependence at low momentum [7, 8]. The core polarization contributions to the proton and neutron potential may cancel out with each other and thus it modifies only slightly the symmetry potential at low momentum [8]. Therefore inclusion of the core polarization will not al- 
ter our above discussion and conclusion.

FIG. 1: Neutron and proton BHF mean fields at different isospin asymmetries for three different nucleon densities

FIG. 2: Isospin splitting the proton and neutron s.p. potentials at $k=0$ : isospin $T=0$ (solid curves) channels, $T=1$ (dashed curves) channels, $S D$ tensor channel (squares).

\section{Effective mass}

The nucleon effective mass $m^{*}$ stems from the nonlocal nature of the s.p. potential felt by a nucleon propagating in nuclear medium. It is determined by the slope of the real part of the on-shell self-energy $U\left(k, \epsilon_{k}\right)=\operatorname{Re} \Sigma\left(k, \epsilon_{k}\right)$ in momentum space, i.e.

$$
\frac{m^{*}}{m}=1-\frac{\mathrm{d} U\left(k, \epsilon_{k}\right)}{\mathrm{d} \epsilon_{k}} .
$$

The effective mass itself is momentum dependent, but usually its value at the Fermi momentum is considered (hereafter we only discuss the latter). The s.p. energy is determined by the following momentum-energy relation,

$$
\epsilon_{k}=\frac{k^{2}}{2 m}+\operatorname{Re} \Sigma\left(k, \epsilon_{k}\right)
$$

for a given approximation of the self-energy. It is clear that the effective mass arises from both the momentum and energy dependence of the microscopic s.p. potential. It is a different physical quantity from the Dirac effective mass associated to the medium modification of the Dirac spinor, which in fact is due to the self-consistent requirement in the relativistic Dirac-Brueckner approach as it has been clarified in Refs. 9, 22 and discussed afterwards.

In isospin asymmetric nuclear matter since the momentum-dependence of the neutron s.p. potential is different from that of the proton one, the common value of the neutron and proton effective mass is expected to split into two branches as a function of isospin asymmetry parameter. The BHF result with the TBF contribution is displayed in Fig. 3]. Two main features are

FIG. 3: Isospin splitting of the effective mass from a Brueckner calculation with three-body force.

displayed by the results: the linear dependence on the asymmetry parameter $\beta$ extended to the whole asymmetry range, which is a well-known effect [21], and the isospin splitting with $m_{n}^{*}>m_{p}^{*}$ in neutron-rich matter. The latter property is related to the increasing (decreasing) slope of the proton (neutron) potential as discussed in the preceding subsection, but it has not yet received a general consensus. In microscopic theories such as the BHF method and the DBHF approach, the predicted neutron-proton effective mass splitting is such that $m_{n}^{*}>m_{p}^{*}$ at Fermi surface in neutronrich matter. However, within phenomenological models some parametrizations of the extended Skryme-like effective interaction may lead to an opposite isospin splitting [12, 23]. A complete settlement of such a controversy will be one of the most important goals of isospin observables such as the neutron-proton differential flow and the $\pi^{-} / \pi^{+}$ratio in $\mathrm{HIC}$ induced by radioactive beams.

In order to get a deeper theoretical insight into the neutron-to-proton splitting of the effective mass, we want to see how it comes out within the Brueckner many-body theory.

FIG. 4: Neutron and proton mass vs. asymmetry: separate contributions from the E-mass and k-mass in units of the bare nucleon mass. The baryonic density is $\rho=0.17 \mathrm{fm}^{-3}$

The off-shell values of the self-energy $\Sigma(k, \epsilon)$ depend separately on the energy and momentum and, as a consequence, following Mahaux et al. [7], the effective mass can be split into a product of the two contributions:

$$
\frac{m^{*}}{m}=\frac{m_{e}}{m} \frac{m_{k}}{m},
$$

where

$$
\begin{gathered}
m_{e}(k)=m\left[1-\frac{\partial \Sigma(k, \epsilon)}{\partial \epsilon}\right]_{\epsilon=\epsilon_{k}}, \\
m_{k}(k)=m\left[1+\frac{m}{k} \frac{\partial \Sigma(k, \epsilon)}{\partial k}\right]_{\epsilon=\epsilon_{k}}^{-1} .
\end{gathered}
$$

The $k$-mass $m_{k}$ is related to the nonlocality of the microscopic mean field in $r$-space. If the self-energy is energy independent (static limit), then $m_{e}=m$ and the $k$-mass is equal to the effective mass $m^{*}$. The $e$ mass describes the nonlocality in time and is related to the quasi-particle strength by $m_{e}(k)=m / Z(k)$, which gives the discontinuity of the momentum distribution at the Fermi surface, and measures the amount of correlations included in the considered approximation. The two components of the effective mass for protons and neutrons are plotted in Fig. 4. It is seen that the isospin splitting is opposite for the two effective masses $m_{k}$ and $m_{e}$. In neutron-rich matter the neutron $k$-mass becomes larger than the proton one, while the neutronproton e-mass splitting is reversed. Although the absolute strengths of the splitting for the two masses are about the same, the relative splitting of the $k$-mass is 
much more pronounced than that of the $e$-mass due to the smaller value of the $k$-mass. As a consequence, the $k$-mass predominates the isospin splitting of the total effective mass $m^{*}$ and leads to the result $m_{n}^{*}>m_{p}^{*}$ in neutron-rich nuclear matter. This result indicates that the effective mass splitting is dominated by the nonlocality of the microscopic s.p. potentials in spatial space.

The effective mass so far discussed is also named Schrödinger mass to distinguish from the Dirac mass appearing in the relativistic mean field theory (RMT) and the relativistic DBHF approach [22, 24, 25]. The Dirac effective mass stems from the self-consistency requirement between the s.p. wave function and the s.p. spectrum of the Dirac spinor which is dressed in nuclear medium, and has no any counterpart in the non-relativistic limit. As discussed in Ref. [24], the Dirac mass can be traced back to the effect of the virtual nucleon-antinucleon pair excitations, i.e., the inmedium positive-energy spinor is an admixture of the free negative- and positive-energy spinors. In Ref. 22], the origin of different effective masses defined in literatures has been discussed in more details. It is shown that in the relativistic framework, an nonrelativistictype of effective mass can be introduced based on the corresponding Schrödinger equivalent s.p. potential and it can be compared to the empirical value extracted from analyses in the framework of the nonrelativistic optical and shell models. Investigations for determining the relativistic effective mass in terms of the momentum dependence of the s.p. energies in the DBHF framework have been made for symmetric nuclear matter in Ref. [25]. Very recently, Van Dalen et al. 26] have investigated the isospin splitting of the nonrelativistic-type effective mass obtained from the Schrödinger equivalent s.p. potential within the DBHF approach. They find that both the dynamical structure (i.e., the momentum and density dependence) and the splitting of the nonrelativistic-type effective mass are satisfactorily consistent with the predictions of the nonrelativistic BHF approach. The Dirac mass in neutron-rich matter shows an opposite isospin splitting of $m_{\mathrm{D}, \mathrm{n}}^{*}<m_{\mathrm{D}, \mathrm{p}}^{*}$, indicating that the virtual pair effect on a neutron spinor becomes stronger as the matter goes to neutron-richer. The nonrelativistic-type of effective mass in neutron-rich matter derived from the RMT displays the same behavior of isospin splitting as the Dirac mass, which is due to the fact that the nonlocal structure of the self-energy is neglected in the RMT as discussed in Ref. [26].

\section{SYMMETRY POTENTIAL AND OPTICAL-MODEL POTENTIAL}

The microscopic self-energy in the BHF approach is nonlocal in space-time coordinates and thus depends on both momentum and energy. When evaluated on the energy shell, it corresponds to the empirical optical model potential 22] :

$$
U_{\text {opt }}(E)=\Sigma(k(E), E),
$$

where $E$ is the incident energy, and $\Sigma(k(E), E)$ the on-shell self-energy. The momentum $k=k(E)$ is determined by the mass-shell relation

$$
E=\frac{\hbar^{2} k(E)^{2}}{2 m}+\Sigma(k(E), E)
$$

FIG. 5: BHF isospin symmetry potential vs. momentum $k$ for three values of density.

The isovector part of the s.p. potential, which drives the isospin splitting of the nucleon mean field in asymmetric nuclear matter, is linearly decreasing with $\beta$ and thus the symmetry potential can be defined as

$$
U_{\mathrm{sym}}=\frac{U_{n}-U_{p}}{2 \beta}
$$

where $U_{n}$ and $U_{p}$ are the s.p. potentials felt by a neutron and proton in nuclear medium, respectively. In Fig. [5 the BHF symmetry potential $U_{\text {sym }}$ is displayed as a function of momentum $k$ for three densities and several isospin asymmetries. The nucleon-nucleus scattering is not influenced so much by the core polarization and it can be neglected. It is seen that the symmetry potential depends strongly upon both density and momentum. Above the Fermi surface $U_{\text {sym }}$ decreases rapidly as a function of momentum and saturates at high enough momenta. In the momentum region relevant to the intermediate HIC up to a beam energy about $300 \mathrm{MeV}$ per nucleon $U_{\text {sym }}$ is positive, implying that its effect is repulsive on neutrons and attractive on protons. At higher densities the repulsion (attraction) on neutrons (protons) becomes stronger. In Fig.5 it is also shown that $U_{\text {sym }}$ is almost independent of the isospin asymmetry $\beta$, which indicates that the linear dependence of the neutron and proton s.p. potentials on $\beta$ persists at any energy and thus it provides a microscopic support of the empirical assumption of the Lane potential [27].

Experimentally the strength of the Lane potential and its momentum dependence can be extracted from the nucleon-nucleus scattering data and/or $(\mathrm{p}, \mathrm{n})$ charge exchange reactions. Earlier optical model analyses of the experimental data with incident energies between 7 and $100 \mathrm{MeV}$ indicate that the $\left(U_{n}-U_{p}\right) / 2 \beta$ at normal nuclear matter density has a value of about $28 \pm 6 \mathrm{MeV}$ at $k=0$ and decreases as a function of incident energy with a slope between 0.1 to 0.2 [28, 29].

The Lane potential is represented by the dashed area, as results extracted from the experimental data of nucleon-nucleus scattering based on the optical potential model 28]. This area is crossed by both the BHF symmetry potential for $\beta=0.2$ and the bulk contribution of the empirical one from Ref. [30] discussed later. 
The predicted strength of the isospin splitting at $k=0$ is about $25 \mathrm{MeV}$ in good agreement with the empirical value $22 \sim 34 \mathrm{MeV}$, extracted from the experimental data of nucleon-nucleus scattering based on the optical potential model [28, 30].

FIG. 6: Comparison among different symmetry potentials: the dashed area is the Lane potential $a-b E_{k i n}$ with $a=22-$ $34 \mathrm{MeV}$ and $b=0.1-0.2$ (see Ref. 31] for more details), the dashed line is the BHF result for asymmetric nuclear matter with $\rho=0.16 \mathrm{fm}^{-3}$ and $\beta=0.2$ and the solid curve is from empirical parametrization of nucleon- ${ }^{208} \mathrm{~Pb}$ scattering [30].

A recent investigation [30] on a broad range of mass $(24 \leq A \leq 209)$ and incident energy $(1 \mathrm{KeV} \leq E \leq$ $200 \mathrm{MeV}$ ) provides a new parametrization of the optical model potential in terms of volume, surface, spinorbit and Coulomb contributions. Therefore, it should be more suitable than the one of Fig. [6] for a comparison with the microscopic potential of nuclear matter, including the density, isospin and energy dependence. Since a direct comparison does not take into account the density variation in the nuclear surface, we have folded the nuclear-matter mean field with the density profile of different nuclei obtained from a ThomasFermi approximation 20]. So doing we can compare the folded mean field with the optical potential as a function of $\beta=(N-Z) / A$. The results are plotted in Fig. 7 for incident energy equal to the Fermi energy. The theoretical predictions slightly overestimate the empirical ones, but the isospin shift turns out to be nicely reproduced. Actually the Thomas-Fermi approximation works better with heavy nuclei for which the comparison looks much better. The momentum dependent optical-model potential of Ref. 30 gets out of the dashed area at low energy, indicating that some uncertainties still affect the optical-model parametrizations.

FIG. 7: Isospin shift of empirical optical potential at the Fermi energy (volume term only) for several nuclei(symbols) in comparison with the theoretical nuclear-matter BHF predictions (big triangles joined by dashed lines). Small symbols are the Optical potential fit by Koning and Delaroche (NPA 2003).

FIG. 8: Comparison of the BHF symmetry potential with other predictions (see text).

At high density and energy one may have to rely on the scarce information from HIC induced by high energy radioactive beams. In this regard, it is interesting to mention that isospin diffusion has been found to be rather sensitive to the momentum-dependence of $U_{\text {sym }}$ [14]. Up to now only the phenomenological parametrizations of the momentum-dependent symmetry potential has been adopted in the dynamical simulations of heavy ion collisions, therefore it is instructive to make a comparison between the present microscopic symmetry potential with the phenomenological ones 12]. In Fig. 8 is plotted the symmetry potentials versus momentum for three values of density $\rho=0.085,0.17$ and $0.34 \mathrm{fm}^{-3}$. In the figure the curves with filled symbols are the results from the BHF calculations, repeated in the four panels, while the curves with open symbols are the phenomenological ones of Ref. 12] and different panels correspond to different parametrizations. It is clear from the figure that the microscopic $U_{\text {sym }}$ shows a remarkably different behavior from the phenomenological ones as a function of density and momentum. All of the four phenomenological symmetry potentials drop much faster at high density $\rho=0.34 \mathrm{fm}^{-3}$ as the momentum increases as compared to our BHF one. In the two cases of the $\operatorname{GBD}(0)$ and $\operatorname{GBD}(1)$ parametrization, the deviation from the $\mathrm{BHF}$ prediction is especially large in the whole density and momentum regions considered here. At the normal nuclear density, the momentum dependence of the Gogny and MDI(0) parametrization is closer to our microscopic one, but the Gogny $U_{\text {sym }}$ presents an opposite density dependence and discrepant dramatically with the microscopic one at high densities. For example, at $\rho=0.34 \mathrm{fm}^{-3}$ the Gogny $U_{\text {sym }}$ is attractive (repulsive) for neutrons (protons), while the BHF one is repulsive (attractive) for neutrons (protons) up to $k \simeq 4 \mathrm{fm}^{-1}$. Even the $\mathrm{MDI}(0)$ parametrization which is closest to our microscopic prediction, turns out to become quite different at high densities.

As the last point we discuss the effect of the rearrangement contribution of the TBF. Due to the density dependence of the effective force $\tilde{v}$, the TBF provides an extra repulsive contribution $\Sigma_{t b f}$ (see Eq.(3) to both the proton and neutron s.p. potentials. At high density this contribution is expected to be strongly momentum dependent and may affect considerably the high momentum components of the fragmentation residues in HIC. We compare our calculated results with the parametrization of the optical potential used in the transport-model simulations of elliptic flows in central HIC [32], where high densities are reached. The potentials in symmetric nuclear matter at $\rho=0.3 \mathrm{fm}^{-3}$ are shown in Fig 9 , where the line with full squares is the one of Ref. 32 which has been shown to describe the observed elliptic flow data fairly well. It is clear from the figure that the BHF potential without the TBF is too attractive, especially at high densities, as compared to the one proposed in Ref. [32], and its momentum dependence at high momenta turns out to be too weak [32] for describing the experimental elliptic flow data. Inclusion of the TBF effect only via the $G$-matrix, i.e., inclusion of the TBF effect in the first two terms of Eq.(3), weakens the dependence of the 
s.p potential on momentum[4]. It is seen from Fig. 9 that the rearrangement contribution of the TBF, i.e., the third term $\Sigma_{t b f}$ of Eq.(3), provides a strongly extra repulsion to the optical potential and improves remarkably the agreement between our microscopic potential and the parametrized one of Ref. 32. We find that the TBF rearrangement leads to also a strong momentum dependence at high densities. For instance at $\rho=0.3$ $\mathrm{fm}^{-3}$ the extra term turns out to be $\Delta U=5.68+6.84 k^{2}$ in units of $\mathrm{MeV}$.

To study the effect of the TBF rearrangement on the isospin symmetry potential, we report in Fig. 10 the results in comparison with the recent DBHF predictions from Refs. 9, 10]. Therein it is displayed the neutron s.p. potential (left-upper panel), the proton s.p. potential (left-lower panel) and the symmetry potential (right panel) in neutron-rich matter with $\beta=0.4$ for both cases with and without including the $\Sigma_{t b f}$ term. It is seen that the BHF neutron and proton s.p. potentials without the $\Sigma_{t b f}$ term are much more attractive than the DBHF ones. Inclusion of the $\Sigma_{t b f}$ term leads to a strong enhancement of the repulsion of both the proton and neutron s.p. potentials and reduces substantially the disagreement between the s.p. potentials predicted by the nonrelativistic BHF and the relativistic DBHF approaches. It is shown in the right panel of Fig. 10] that the effect of the $\Sigma_{t b f}$ term on the isospin symmetry potential is very weak, indicting that the contributions of the $\Sigma_{t b f}$ term to the neutron and proton potentials cancel out in a wide momentum range with each other to a large extent. In both cases with and without the $\Sigma_{t b f}$ term, the isospin symmetry potentials obtained by the BHF approach display an overall agreement with those by the DBHF approach. At high momentum our symmetry potential is slight lower than the DBHF ones. The difference between the two DBHF calculations may be attributed to the different methods adopted to extract the selfenergy as discussed in Ref. 34] where it is shown that the determination of the nucleon selfenergy in the DBHF framework is still affected by some uncertainties.

FIG. 9: Optical potential in nuclear matter as a function of nucleon energy at density $\rho=0.3 \mathrm{fm}^{-3}$ from the BHF calculation including all the three terms of Eq 3 with the Argonne $V_{18}$ interaction plus TBF (solid line). The squares are from Ref. 32]. The circles are from the earlier BHF calculation without three-body force [33].

\section{SUMMARY AND CONCLUSIONS}

In summary, we have investigated the momentum and density dependence of the symmetry potential and discussed the origin of the neutron-proton effective
FIG. 10: Comparison of the BHF symmetry potential with and without the $\Sigma_{t b f}$ contribution with Dirac-Brueckner predictions. The curve with full circles is from Ref. [10], the one with full squares is from Ref. [9]

mass splitting in neutron-rich nuclear matter within the framework of the Brueckner theory.

We have found that the isospin behavior of the momentum dependent neutron and proton s.p. potentials can be traced back to the effect of the $S D$ tensor component of the NN interaction and, consequently, the neutron-proton effective mass splitting is essentially determined by the intrinsic properties of the NN interaction. The obtained neutron-proton effective mass splitting in neutron-rich matter is $m_{n}^{*}>m_{p}^{*}$ in good agreement with the recent predictions by the non relativistic limit of DBHF approach [26]. The isospin splitting $m_{n}^{*}>m_{p}^{*}$ is shown to stem from the splitting of the $k$-mass, i.e., from the spatial nonlocality of the microscopic neutron and proton s.p. potentials.

It turns out that the predicted symmetry potential depends sensitively on density and momentum, but almost independent of the isospin asymmetry (Lane ansatz). In the energy and density regions most relevant for the nucleus-nucleus scattering up to an incident energy of about $300 \mathrm{MeV}$ per nucleon, our microscopic symmetry potential is repulsive for neutrons and attractive for protons, and its strength becomes smaller as momentum increases for a fixed density. A satisfactory support to the microscopic predictions is provided by a recent optical model parametrizations of nucleonnucleus scattering [30]. In dense nuclear matter, which can be probed in HIC at intermediate and high energies, the symmetry potential turns out to become stronger in the high momentum region up to about $4 \mathrm{fm}^{-1}$ as increasing density. At high energy relativistic effects manifest with a strong momentum dependence [26, 32], which can be interpreted as an effect of the TBF. At the present time a comparison with phenomenological Skyrme-like or Gogny predictions of the symmetry potential [31] is quite difficult, and empirical constraints on their parameters are needed to make it useful.

In the BHF approach, the TBF contribution has been included by reducing the TBF to an equivalent effective two-body force. The rearrangement effect due to the density dependence of the equivalent force has been found to provide an extra repulsive contribution to the proton and neutron s.p. potentials, which improves substantially the agreement of our nonrelativistic s.p. potential with the parametrized potential for describing the elliptic flow data 32 and those predicted by the DBHF approach 9, 10]. The TBF rearrangement has been found to affect only slightly affect the isospin symmetry potential due to the cancellation between the 
two contributions to the proton and neutron potentials. Our calculated symmetry potential is shown to be in an overall agreement with the DBHF predictions.

Physical observables which are sensitive to the symmetry potential, including the neutron-to-proton ratio of pre-equilibrium nucleon emission, neutron-to-proton differential flow and isospin diffusion, are expected to provide experimental constraints on the momentum and density dependence of the symmetry potential [31].

\section{ACKNOWLEDGMENT}

We thank Dr. P. Danielewicz for valuable comments. One of us (W. Zuo) acknowledges the warm hospitality extended to him at LNS-INFN (Catania) where this work has been carried out. The work was done within the Asia-Link project'Nuclear Physics and Astrophysics' (CN/ASIA-LINK/008(94791)) granted by the European Commission. The work of W. Zuo was supported in part by the Major Prophase Research Project of Fundamental Research of the Ministry of Science and Technology of China (2002CCB00200), the Chinese Major State Basic Research Development Program (G2000077400), the Knowledge Innovative Project of CAS (KJCX2-SW-N02), and the National Natural Science Foundation of China (10235030). The work of Bao-An Li was supported in part by the US National Science Foundation under grants PHY0243571, PHY0354572 and the NASA-Arkansas Space Grants Consortium Award ASU15154.
[1] A. Cunsolo et al., Nucl. Instrum. and Methods A 481 (2002) 48.

[2] J. Nolen, The Rare Isotope Facility: Overview of the project, Proc. RIA Applications Workshop 2000, Durham, NC, 2000.

[3] H. Q. Song, M. Baldo, G. Giansiracusa, and U. Lombardo, Phys. Rev. Lett. 81, 1584(1998); M. Baldo, A. Fiasconaro, H. Q. Song, G. Giansiracusa, and U. Lombardo, Phys. Rev. C 65, 017303 (2002).

[4] W. Zuo, A. Lejeune, U. Lombardo, and J. -F. Mathiot, Nucl. Phys. A 706, 418 (2002).

[5] W. Zuo, A. Lejeune, U. Lombardo, and J. -F. Mathiot, Eur. Phys. J. A 14, 469 (2002).

[6] W. Zuo, C. W. Shen and U. Lombardo, Phys. Rev. C 67, 037301 (2003); U. Lombardo, C. W. Shen, N. Van Giai and W. Zuo, Nucl. Phys. A 722, 532c (2003).

[7] J.P. Jeukenne, A. Lejeune and C. Mahaux, Phys. Rep. 25, 83 (1976).

[8] W. Zuo, I. Bombaci, and U. Lombardo, Phys. Rev. C 60, 024605 (1999).

[9] E.N.E. van Dalen, C. Fuchs and A. Faessler, Nucl. Phys. A 744, 227 (2004).

[10] F. Sammarruca, W. Barredo, and P. Krastev, arXiv: nucl-th/0411053 D. Alonso and F. Sammarruca, Phys. Rev. C 67, 054301 (2003).

[11] B. A. Li, Phys. Rev. Lett. 88, 192702 (2002).

[12] C. B. Das, S. Das Gupta, C. Gale and B. A. Li, Phys. Rev. C 67, 034611 (2003).

[13] B. A. Li, C. B. Das, S. Das Gupta, and C. Gale, Phys. Rev. C 69, 011603 (2004); Nucl. Phys. A 735, 563 (2004).

[14] L.W. Chen, C.M. Ko and Bao-An Li, Phys. Rev. Lett. 94, 32701 (2005).

[15] B. A. Li, G. C. Yong and W. Zuo, Phys. Rev. C 71, 014608 (2005); ibid, C71, 044604 (2005).

[16] R. B. Wiringa, V. G. J. Stoks, and R. Schialla, Phys.
Rev. C 51, 38(1995).

[17] P. Grangé, A. Lejeune, M. Martzolff, and J.-F.Mathiot, Phys. Rev. C40, 1040(1989).

[18] R. Machleidt, Adv. Nucl. Phys. 16, 189 (1989).

[19] R. Sartor, in Nuclear Methods and the Nuclear Equation of State, Ed. M. Baldo, (World Scientific, Singapore, 1999), Chapt.6, P.1.

[20] P. Ring, and P. Schuck, The Nuclear Many-Body Problem, ( Springer-Verlag, New York, 1980 ).

[21] I. Bombaci and U. Lombardo, Phys. Rev. C44, 1892 (1991).

[22] M. Jaminon, and C. Mahaux, Phys. Rev. C 40, 354 (1989).

[23] J. Rizzo, M. Colonna, M. Di Toro and V. Greco, Nucl. Phys. A 732202 (2004).

[24] G.E. Brown, W. Weise, G. Baym, and J. Speth, Comments Nucl. Part. Phys., 17, 39 (1987).

[25] R. Brockmann, and R. Machleidt, Phys. Rev. C 42, 1965 (1990).

[26] E. N. E. van Dalen, C. Fuchs, and A. Faessler, Effective Nucleon Masses in Symmetric and Asymmetric Nuclear Matter, nucl-th/0502064

[27] A. M. Lane, Nucl. Phys. 35676 (1962).

[28] P.E. Hodgson, The Nucleon Optical Model, (World Scientific 1994), page 613-651.

[29] G.W. Hoffmann and W.R. Coker, Phys. Rev. Lett. 29, 227 (1972).

[30] A.J. Koning and J.P. Delaroche, Nucl.Phys. A713, 231 (2003)

[31] Bao-An Li, Phys. Rev.C 69, 064602 (2004).

[32] P. Danielewicz, Nucl. Phys. A 673, 375 (2000).

[33] M. Baldo, I. Bombaci, G. Giansiracusa, and U. Lombardo, Phys. Rev. C 40, R491.

[34] L. Sehn, C. Fuchs, and A. Faessler, Phys. Rev. C 56, 216 (1997). 


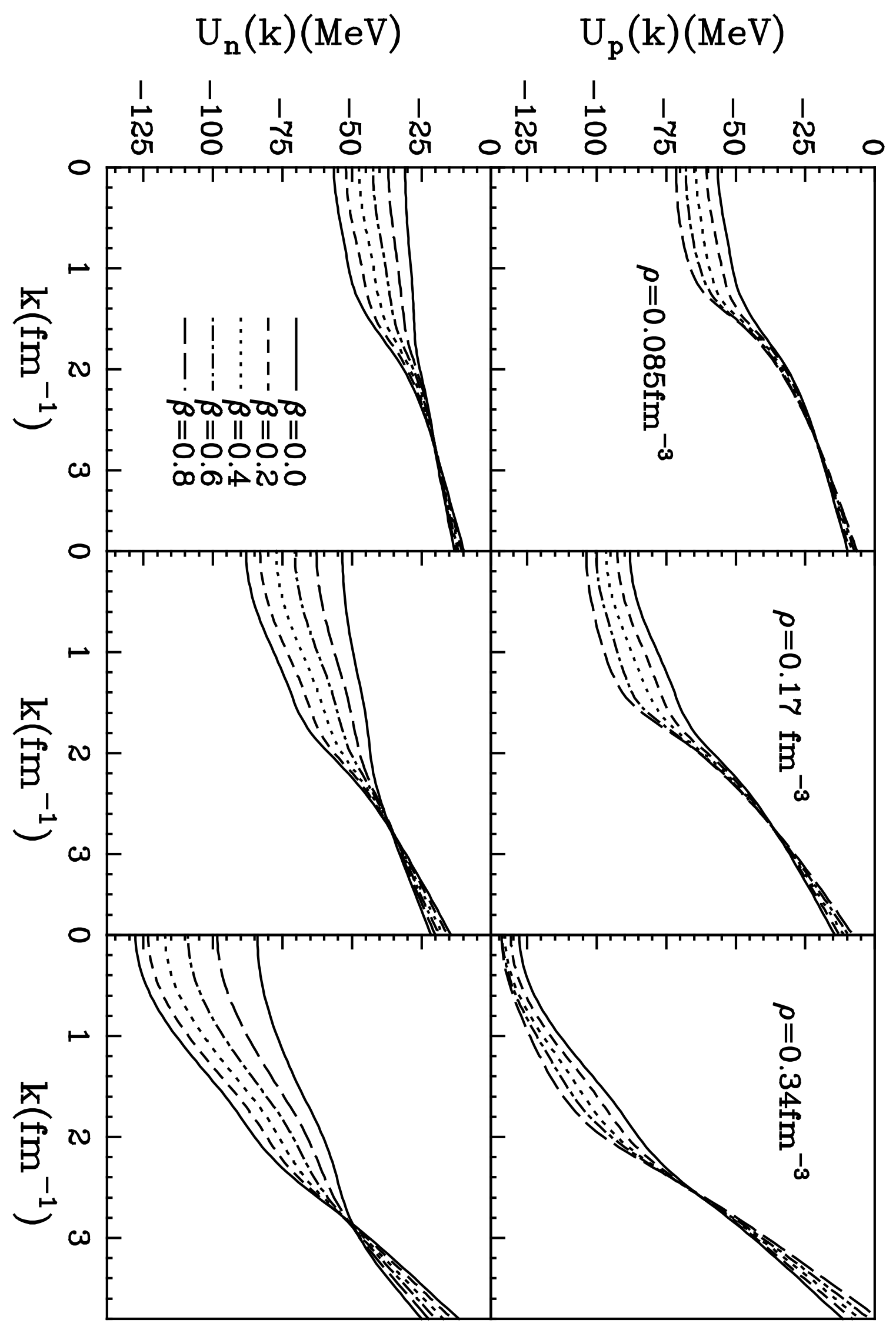




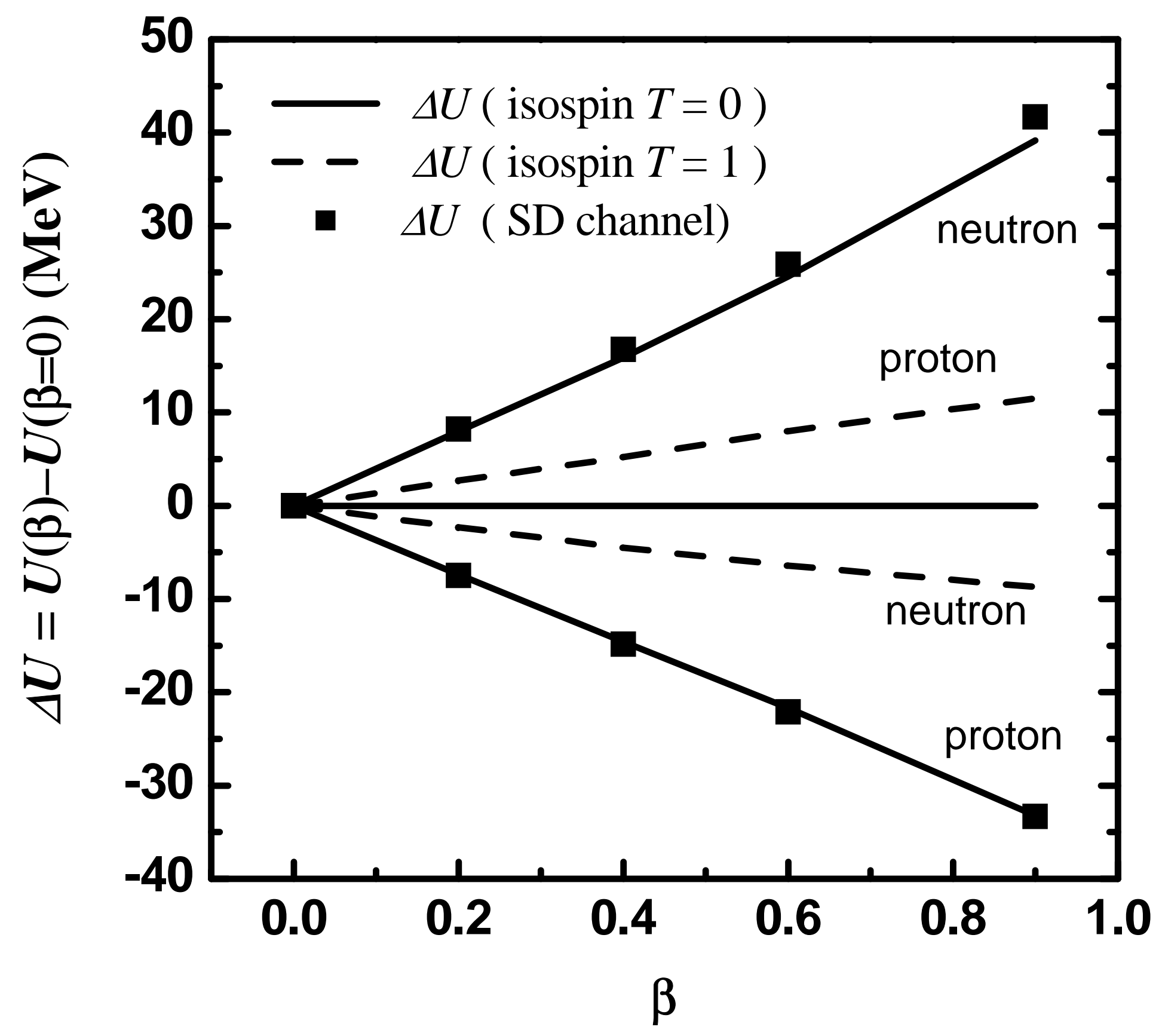




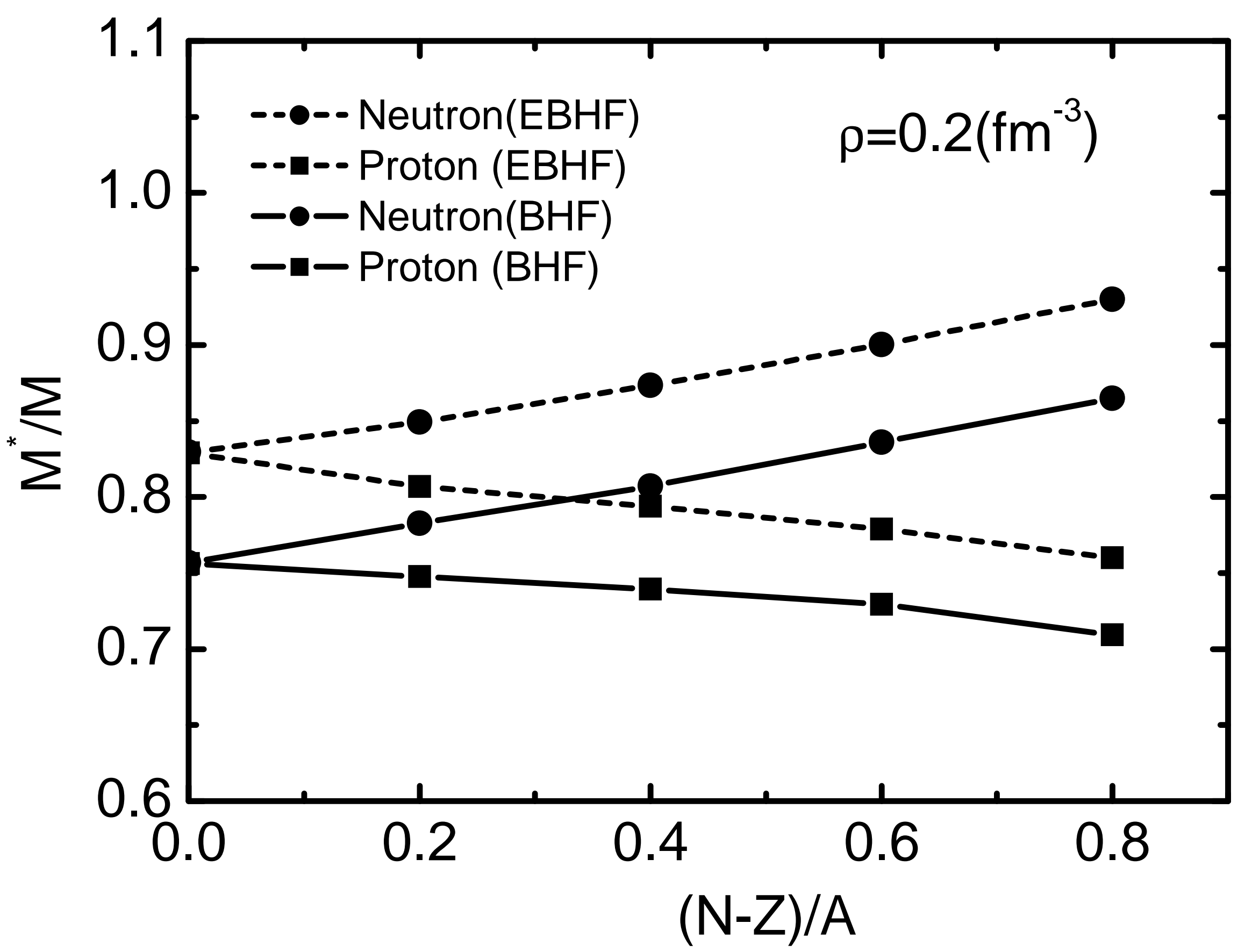




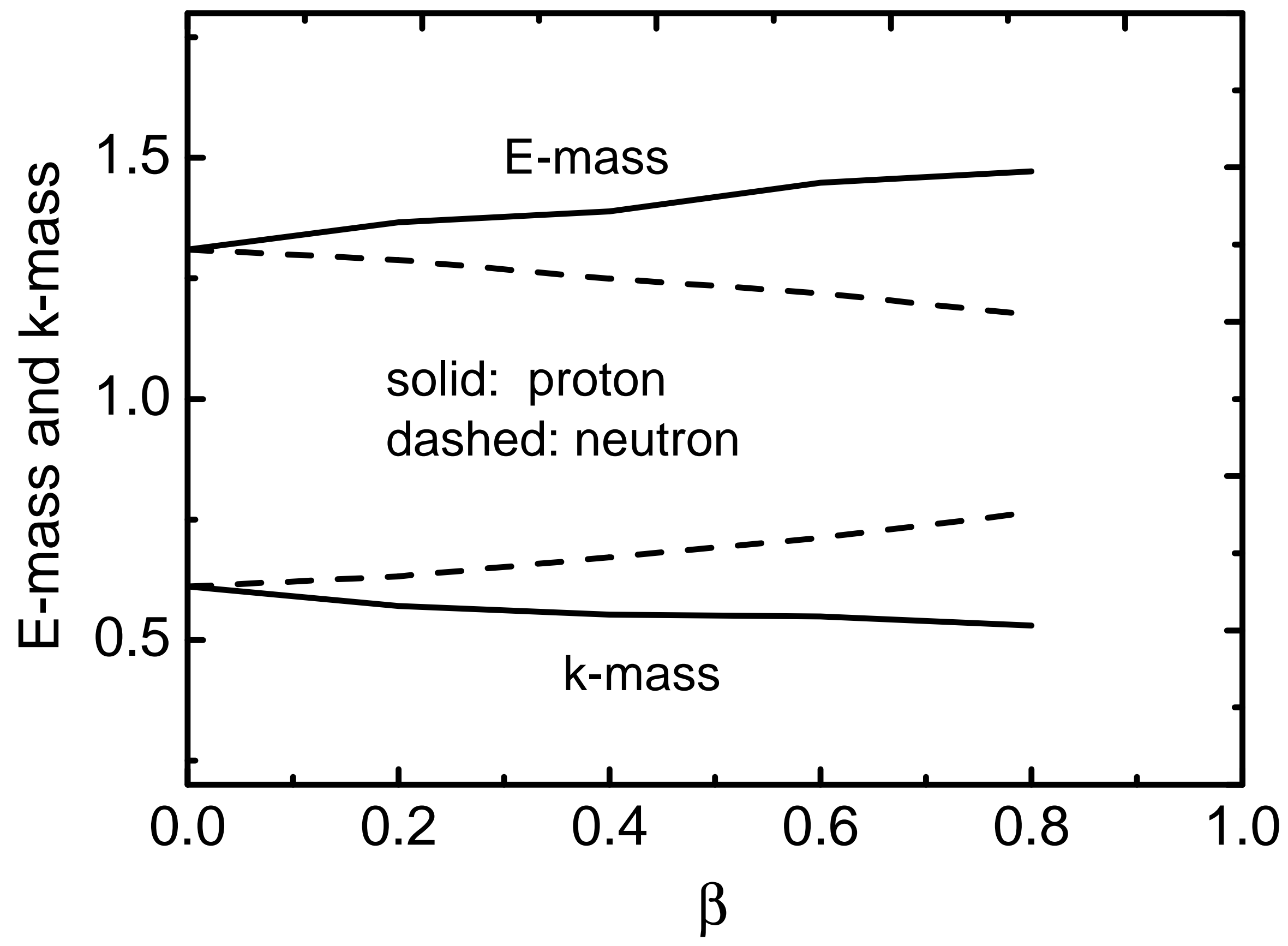




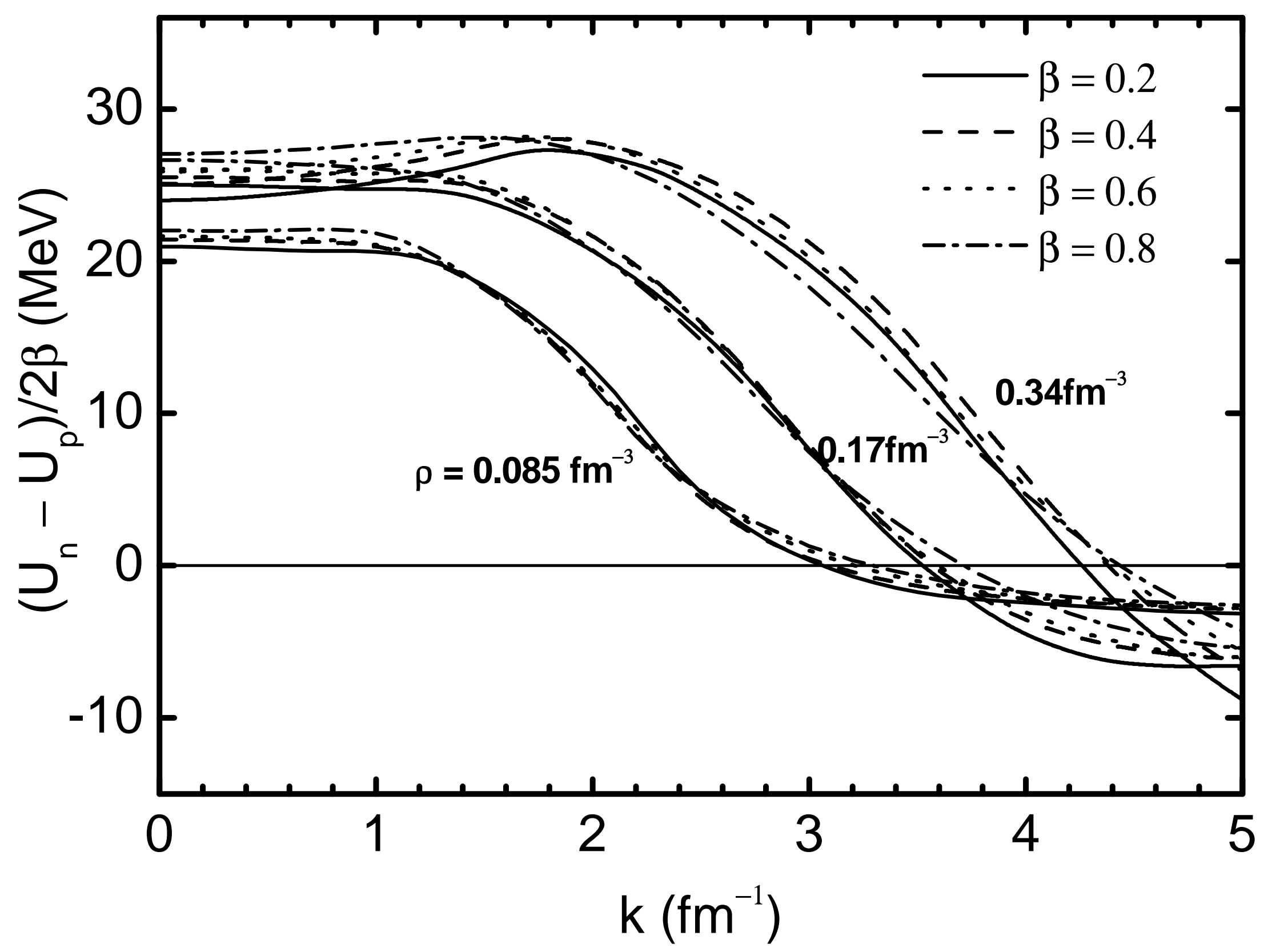




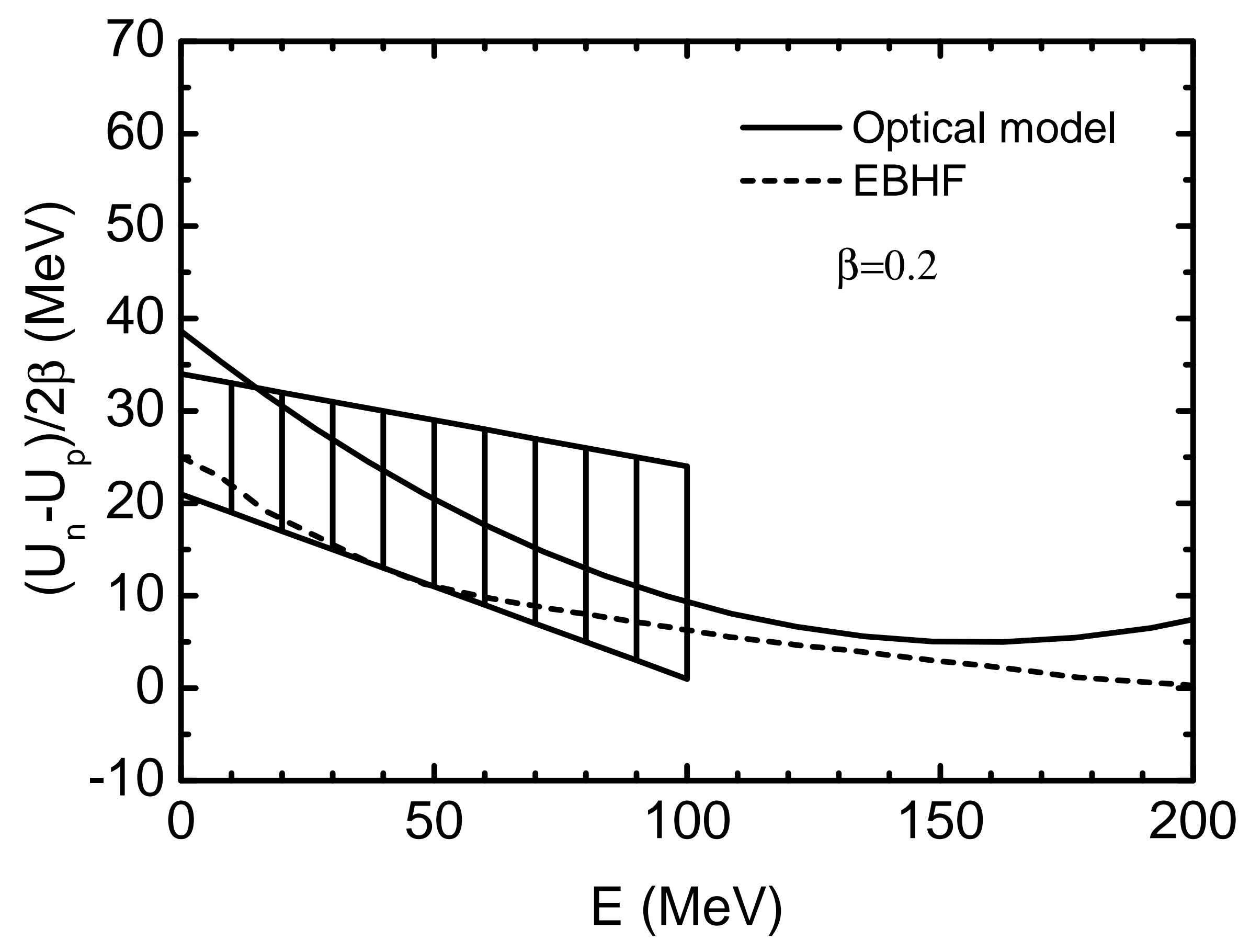




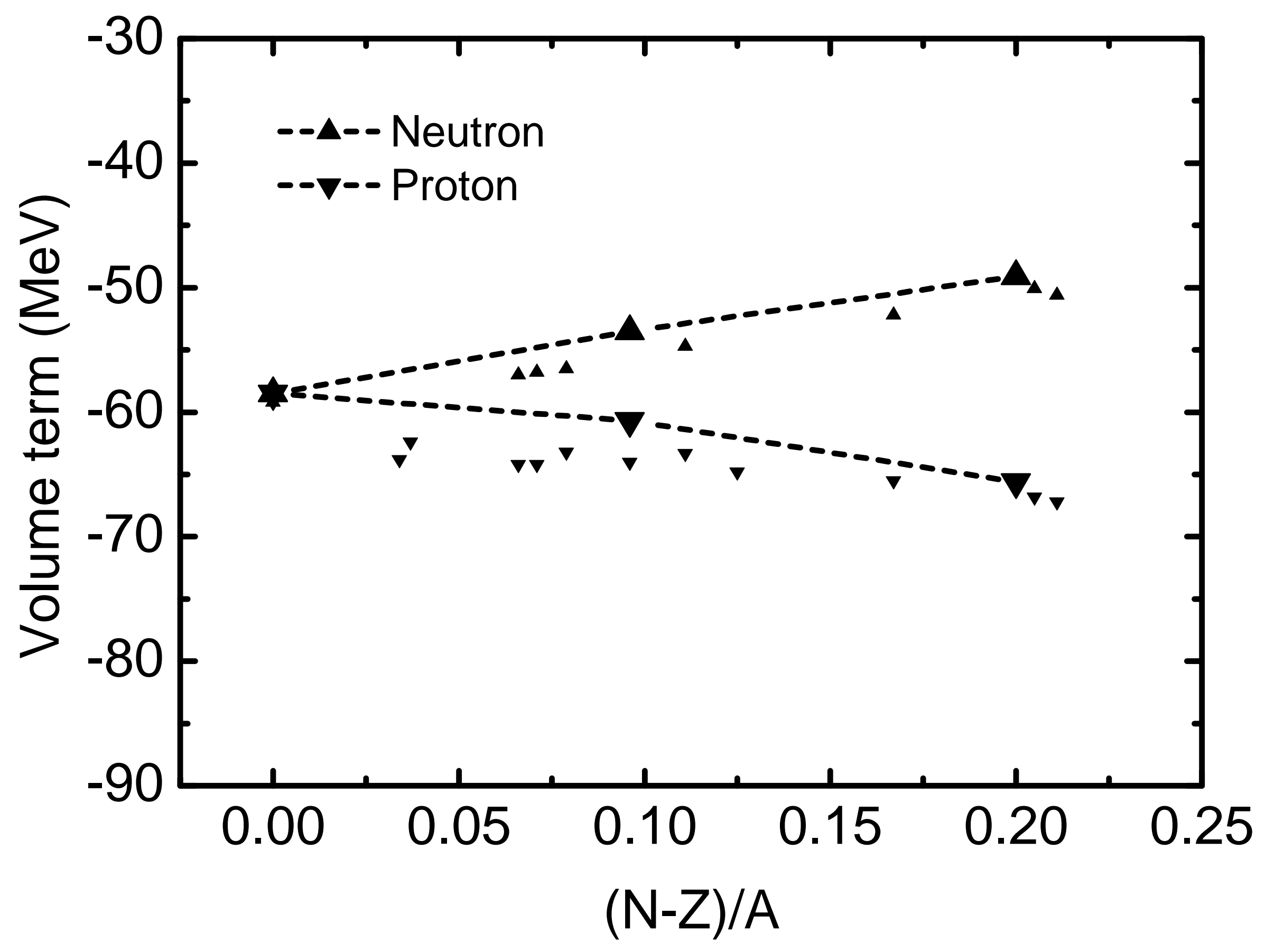




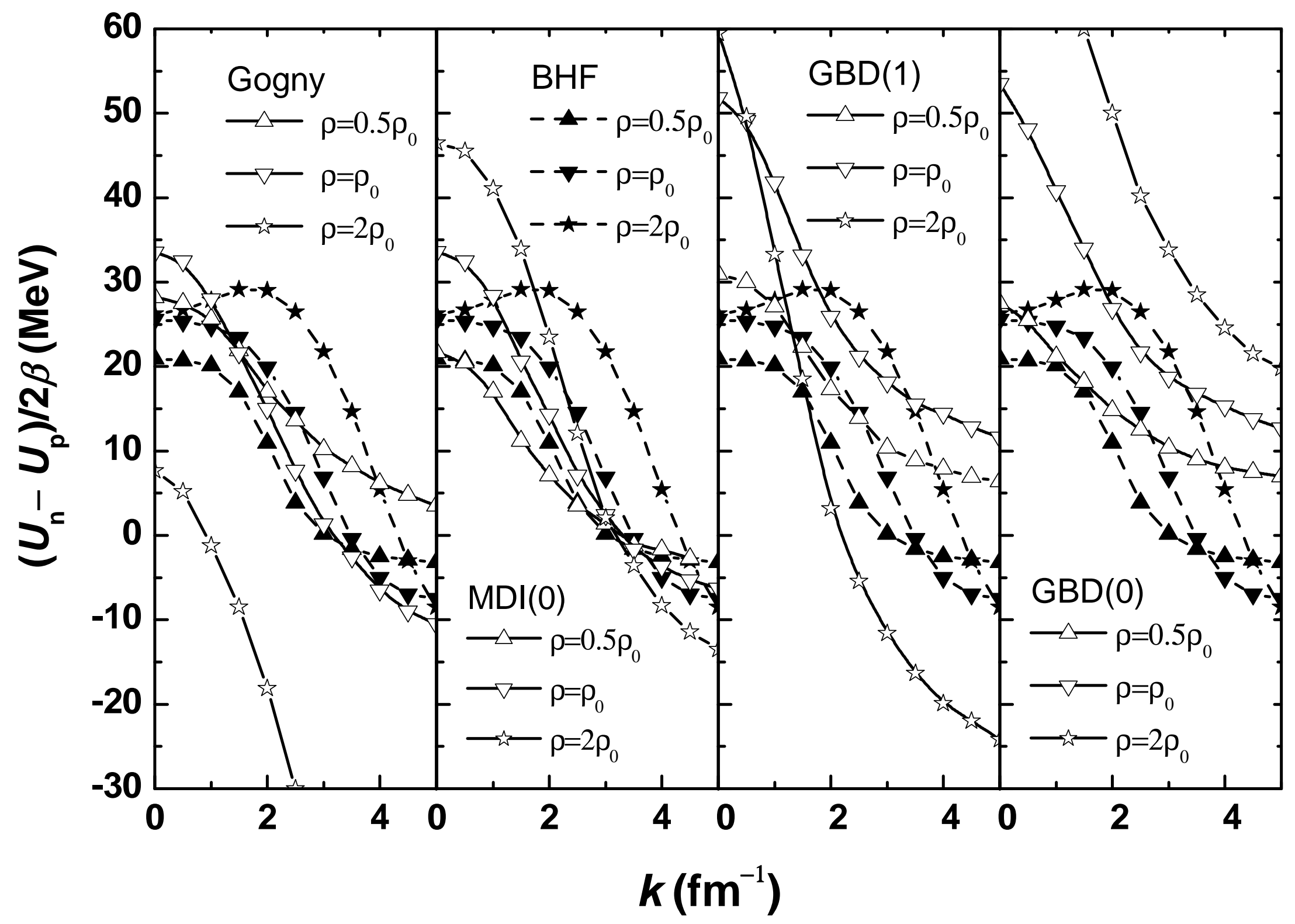




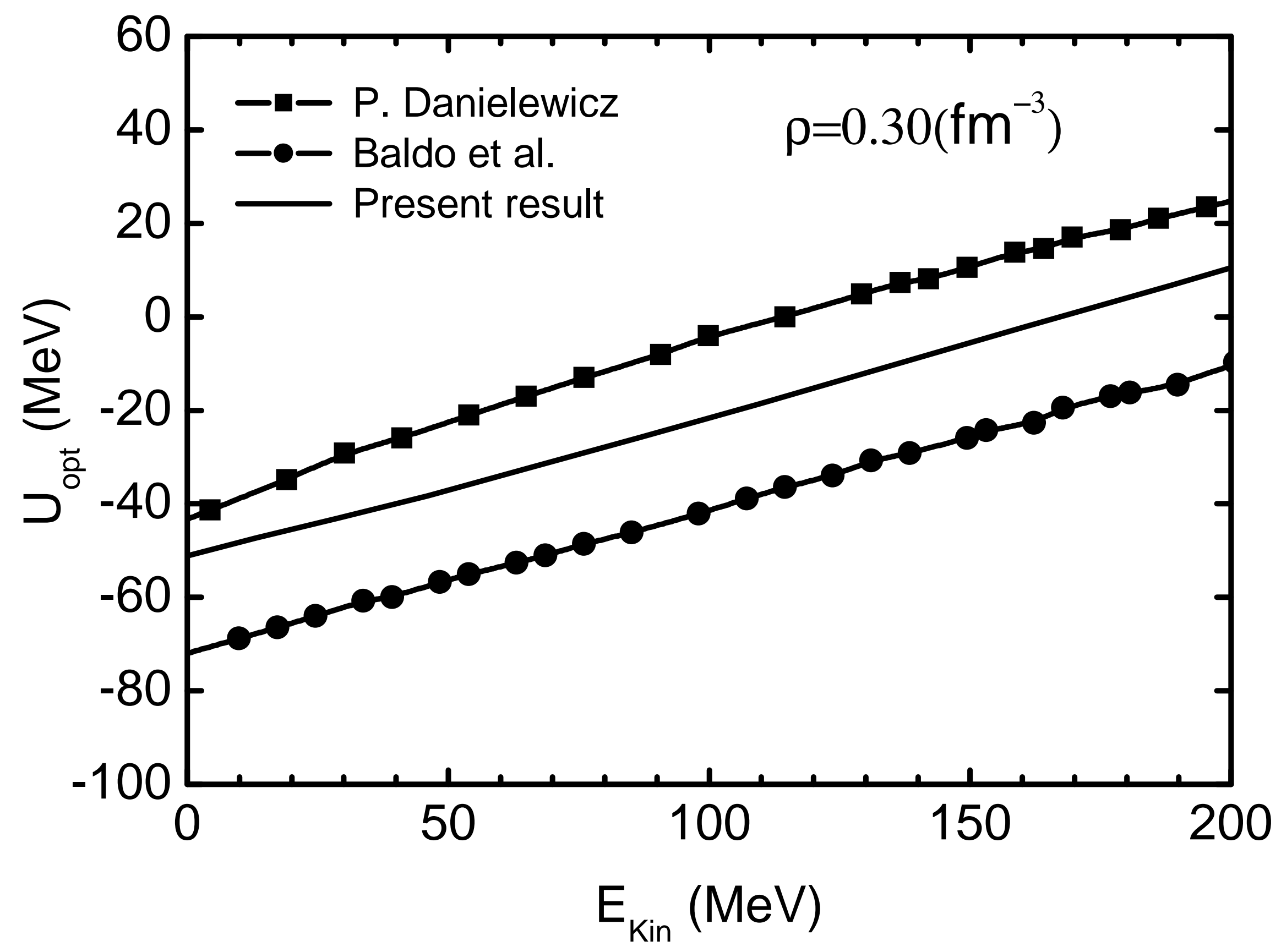



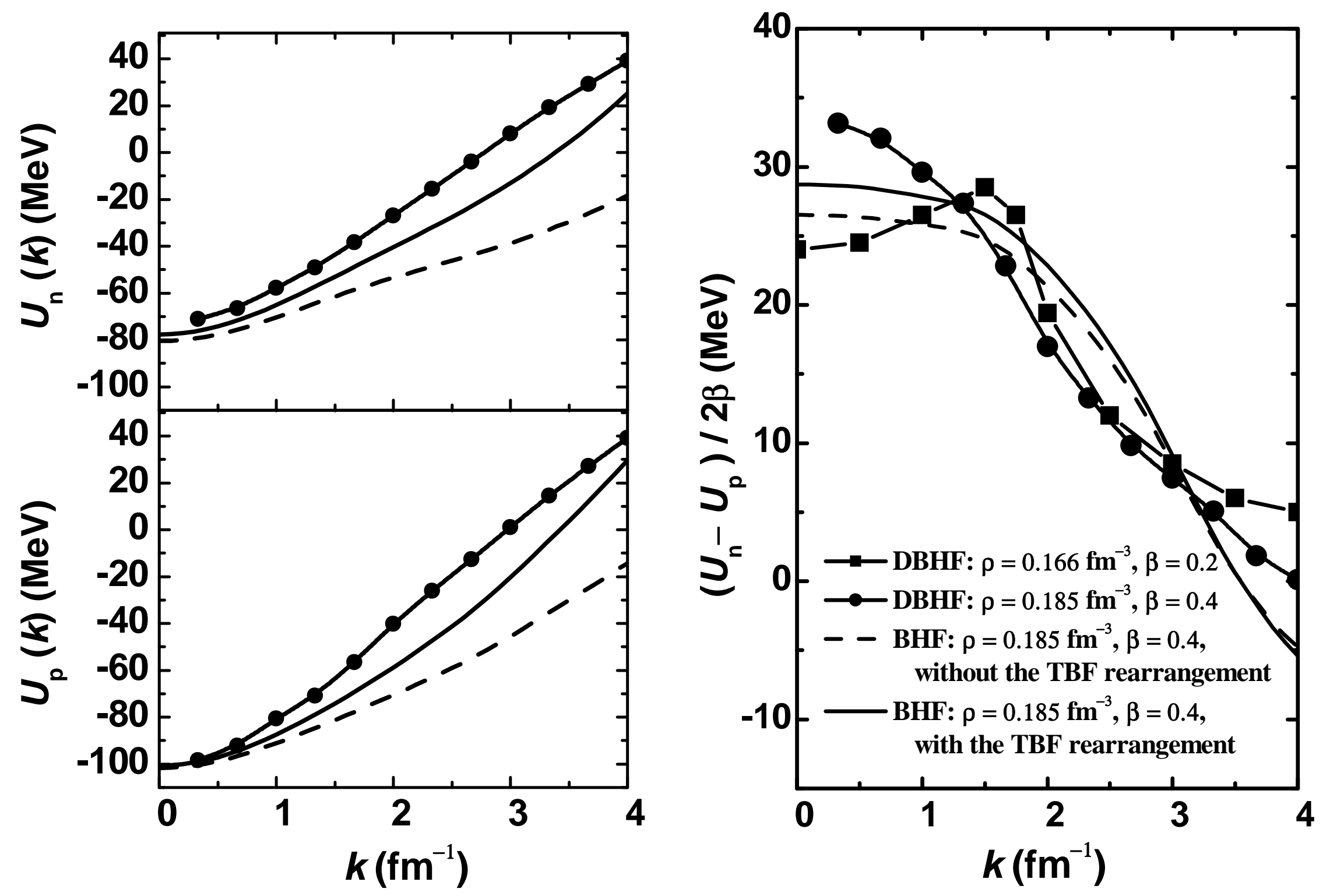